\title{
Género y política: un análisis pertinente
}

\author{
Teresa VALDÉS y \\ Ma. de los Ángeles FERNÁNDEZ
}

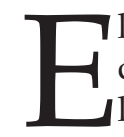

1 siglo XX fue el siglo de las mujeres, como lo revelan las estadísticas y la investigación social en todo el mundo. El cambio en el lugar ocupado por ellas en la sociedad es considerado la principal transformación social, política, económica y cultural vivida en Occidente en ese período. A lo largo de un siglo, las mujeres pasaron de ser consideradas legalmente incapaces y de estar, de preferencia, recluidas a las tareas domésticas de la reproducción, a ser ciudadanas y constituir la mitad o más del electorado, más del $40 \%$ de la fuerza de trabajo así como de la matrícula universitaria, a tener sus propias organizaciones y movimientos sociales y a ocupar posiciones de poder en todas las esferas de la sociedad. Las mujeres son consideradas fundamentales hoy día en las estrategias de desarrollo. En efecto, los gobiernos reunidos en la Organización de las Naciones Unidas han concluido que la igualdad de género y el empoderamiento de las mujeres son imprescindibles para superar la pobreza y alcanzar el pleno desarrollo ${ }^{1}$.

Se trata de cambios dramáticos tanto en la esfera pública como en el ámbito privado, que han alterado un orden que ha tenido vigencia por cuatro mil años o más².

1. En el año 2000, los Estados Partes de Naciones Unidas establecieron una agenda internacional para el nuevo siglo en la Declaración del Milenio. En ella se comprometen a "Promover la igualdad entre los sexos y la autonomía de la mujer como medios eficaces de combatir la pobreza, el hambre y las enfermedades y de estimular un desarrollo verdaderamente sostenible". En 2001, la Asamblea General aprobó los Objetivos de Desarrollo del Milenio, basados en dicha Declaración. El Tercer Objetivo es "Promover la igualdad de los géneros y la autonomía de la mujer" (ONU, 2000).

2. Así lo revelan las investigaciones arqueológica y antropológica. Ver, entre otros, a Lerner (1986), Gimbutas (1995), Miles (1988), Eisler (1990). 
En 1789, la Revolución Francesa había afirmado la libertad, la igualdad y la fraternidad en nombre del universalismo, y si bien selló la exclusión política de las mujeres, instaló las condiciones para su reivindicación (Marques-Pereira, 2003). En efecto, la referencia a la universalidad de los derechos fue el instrumento de lucha contra las desigualdades que animó las posiciones ejemplares del marqués de Condorcet, Olympe de Gouges y Mary Wollstonecraft. En 1790, Condorcet publicó su artículo "L'admission des femmes au droit de cité" en que denunciaba la exclusión política de las mujeres como una violación de los principios de la Declaración de los derechos del hombre y del ciudadano. Un año antes, Olympe de Gouges había redactado la "Declaración de los derechos de la mujer y de la ciudadana" (1789), recordando que la humanidad no era sólo masculina y que el sexismo era la madre de todas las desigualdades. Murió en la guillotina en 1793, acusada de conspirar contra el orden y abandonar las virtudes de su propio sexo. En Inglaterra, Mary Wollstonecraft, en 1790, publicó su artículo "A Vindication of the Rights of Women" en el que insistía en la pertenencia de las mujeres a una misma comunidad humana con los varones, dotados de razón, lo que hacía ilógico e injusto dejar a las mujeres subordinadas a éstos y considerar al hombre como único representante del género humano (Marques-Pereira, 2003). Todos ellos marcaron el camino civilizatorio de la modernidad.

En 1869, el filósofo liberal inglés John Stuart Mill escribió el ensayo “The Subjection of Women" argumentando a favor del acceso de las mujeres al sufragio, a la educación y el empleo. Desarrolló una dura crítica a la dominación masculina en el matrimonio, que dejaba a las mujeres en condición de esclavitud. En cambio, los filósofos que le precedieron concebían la autoridad masculina sobre las mujeres como natural (Aristóteles), como resultado del consentimiento de estas últimas a cambio de protección (Hobbes), como necesaria para conducir el hogar (Locke) o como la consecuencia del amor y la educación de los niños (Rousseau). Mill rechazó la idea misma de naturaleza de la mujer al considerarla un producto eminentemente artificial y defendió la inclusión de las mujeres en la esfera pública y política en nombre del utilitarismo: ¿podía la sociedad rechazar los servicios de una persona competente, considerar incapaz a la mitad de la humanidad sin reconocer sus facultades, por elevadas que fueran?

El universalismo animó a las sufragistas del mundo occidental que, tras arduas luchas, lograron terminar con la cuota del $100 \%$ que se habían autoasignado los varones en los sistemas democráticos, e incorporarse a una ciudadanía activa. Iniciaron entonces el largo camino hacia la "igualdad en derechos y dignidad" de mujeres y hombres, consagrada en 1948 en la Declaración universal de los derechos humanos. En América Latina, recién en 1963 las mujeres de todos los países accedieron al voto, siendo el último país Paraguay (Valdés y Gomáriz, 1995). Para entonces, hacía años que la Comisión Interamericana de 
Mujeres $(\mathrm{CIM})^{3}$ y la Comisión Jurídica de la Mujer de las Naciones Unidas ${ }^{4}$ presionaban a los gobiernos para que igualaran a las mujeres como ciudadanas a través del derecho a elegir y ser elegidas en los más altos cargos de representación popular.

En el caso de Chile, la primera mitad del siglo XX vio la acción concertada de mujeres de todo el país y de los más diversos sectores políticos y sociales que, tras decenios de lucha, lograron la aprobación, por parte de los parlamentarios varones, de la ley que les daba la condición de ciudadanas (1949). Una vez obtenido el derecho a voto, los logros de las chilenas en los partidos políticos y en la institucionalidad democrática, hasta el quiebre de 1973, fueron extremadamente lentos, a pesar de su creciente participación en los actos electorales y en las organizaciones sociales (Valdés y Gomáriz, 1992).

Treinta años después, en condiciones diferentes a las de la lucha sufragista, pero semejantes en cuanto a la exclusión política de la ciudadanía, el país vio surgir otro poderoso movimiento de mujeres. Este movimiento tuvo como elemento central de su agenda política la recuperación de la democracia, se fue articulando crecientemente e incorporó nuevas demandas a la futura democracia: "democracia en el país y en la casa", cambiar las relaciones de poder entre hombres y mujeres en el espacio público, pero también en el ámbito privado, participación con igualdad de oportunidades en el desarrollo y la incorporación de sus demandas en la agenda pública ${ }^{5}$.

En la década del 70 nació la agenda internacional por los derechos de las mujeres y la equidad de género: en 1975 tuvo lugar la Primera Conferencia de Naciones Unidas sobre la Mujer, en Ciudad de México, iniciándose el Decenio para la Mujer (1975-1985), y en 1979, la Asamblea de Naciones Unidas aprobó la Convención sobre la eliminación de todas las formas de discriminación contra la mujer (CEDAW). Esta Convención definió la discriminación contra la mujer, validó las medidas de acción afirmativa para reducir la brecha histórica entre mujeres y hombres y estableció un mecanismo para avanzar en su superación, siendo obligatorio para los países que la ratificaran. En la década del 80 se inició la incorporación de la igualdad de género en la agenda pública en los países del mundo y de América Latina.

En Chile, el rol indiscutido que jugó el movimiento de mujeres en la recuperación de la democracia hizo posible que su agenda política fuera

3. La Comisión Interamericana de Mujeres (CIM) fue creada en 1928 y fue la primera agencia intergubernamental cuyo objetivo fueron los derechos de las mujeres.

4. Creada en 1946.

5. Ver el Pliego de las Mujeres, presentado a la Asamblea de la Civilidad en 1986 e incorporado a la Demanda de Chile, y las Demandas de las Mujeres a la Democracia, publicadas por el Movimiento Feminista en 1988 (Palestro, 1991). 
asumida en gran medida por el gobierno de Patricio Aylwin (1990-1994). Una de sus propuestas fue la creación de un organismo de gobierno especializado en las políticas para la igualdad y responsable del cumplimiento de la CEDAW. El Servicio Nacional de la Mujer (SERNAM), sus primeras políticas y programas, fueron el fruto de estas propuestas elaboradas por mujeres del movimiento ${ }^{6}$. Muchas de ellas se incorporaron al sector público para hacer realidad las políticas que dieran respuesta a las urgentes necesidades de las mujeres. Algunas asumieron cargos políticos y de representación partidaria. El mundo no gubernamental se especializó en distintos temas estableciendo relaciones de colaboración con el Estado (Frohmann y Valdés, 1995).

Desde entonces hemos asistido a una paulatina extensión de la ciudadanía de las mujeres, al lograr transformar sus reivindicaciones en reformas legislativas, en nuevas leyes y en la implementación de políticas públicas destinadas a superar las discriminaciones y alcanzar la equidad (Valdés et al., 2001). Las chilenas han sido parte activa de la comunidad política, han participado en la definición de los problemas comunes a la sociedad y de la forma en que deben ser abordados (Jelin, 1996 y 1997).

La CEDAW, la Declaración de la Conferencia Mundial de Derechos Humanos (Viena, 1993), el Plan de Acción de la Conferencia Internacional sobre la Población y el Desarrollo (El Cairo, 1994), la Convención Interamericana para prevenir, sancionar y erradicar la Violencia contra la Mujer (Belem do Pará, 1994), la Plataforma de Acción Mundial de la IV Conferencia de Naciones Unidas sobre la Mujer (Beijing, 1995) constituyen el marco de derechos humanos de las mujeres que ha orientado la acción de los gobiernos de la Concertación de Partidos por la Democracia.

Las modificaciones introducidas en la legislación a contar de 1991 (ver Anexo 1) y la definición de políticas públicas específicas se han sumado, o han venido a responder a los cambios experimentados por mujeres y hombres en sus vidas. Entre ellos vale destacar la reducción de las tasas de fecundidad y del número deseado de hijos, el aumento de las rupturas matrimoniales, la reducción de la edad de inicio de la vida sexual, la elevación de los niveles educativos y el ingreso masivo a los estudios superiores, la creciente incorporación femenina al mercado de trabajo y la necesidad de conciliar el trabajo y la vida familiar (ver Anexo 2). Los cambios legislativos y las políticas han buscado responder, además, a las discriminaciones sufridas por las mujeres en diversos ámbitos, como son el educativo, el laboral, el acceso a la propiedad y al crédito, la violencia doméstica y sexual, entre otras. Sin embargo, duermen en el Parlamento algunos proyectos de ley estratégicos en materia de equidad y no discriminación, como son la propuesta de ley de cuotas (se han presentado

6. Ver Montecino, Sonia y J. Rossetti (eds.) (1990). 
tres proyectos desde $1997^{7}$ ) y la Ley marco sobre Derechos Sexuales y Reproductivos, que ingresó a la Comisión de Salud de la Cámara de Diputados en octubre de $2000^{8}$.

Todos estos cambios han sido posibles a partir del llamado "triángulo de empoderamiento", constituido por el movimiento de mujeres, femócratas $^{9}$ y feministas políticas (Lycklama et al., 1996). El establecimiento de mecanismos de acción afirmativa en varios partidos políticos de la Concertación (Zavala, 2005) ha sido la antesala de varios de los cambios legislativos mencionados.

Son tan significativos y profundos los cambios que se han operado en nuestra sociedad en cuanto al lugar de las mujeres, que este año una mujer fue elegida Presidenta de la República con un amplio respaldo popular. Su candidatura surgió de la base social y de la Concertación de Partidos por la Democracia, sorprendiendo a los analistas políticos que no terminan de descifrar los procesos que permitieron este nuevo escenario. Se trata de una mujer de características particulares: no sólo socialista, sino además agnóstica y alejada del estereotipo esperable en un país reconocido como católico y machista. Una Presidenta que nombró, tal como lo había prometido en su campaña electoral y en su programa de gobierno, un gabinete ministerial paritario: igual número de mujeres y de hombres. Otro tanto hizo con los gobiernos regionales y provinciales.

Su elección se produce tras un gobierno exitoso de la misma coalición, presidido por otro socialista, Ricardo Lagos, éxito que no logró esconder la profunda crisis que aqueja a los partidos políticos en general. Es el "malestar" con la política, en el decir de Norbert Lechner (2002), que surge de la crisis de los mapas ideológicos, cuando los partidos políticos no logran ofrecer a la ciudadanía pautas de orientación capaces de arti-

7. 1) Boletín Legislativo $\mathrm{N}^{\mathrm{o}}$ 1994-07. Moción de los/as diputados/as Mariana Aylwin O., Isabel Allende B., Camilo Escalona M., Fanny Pollarolo V., Romy Rebolledo L., Andrés Palma I., Martita Wörner T., Víctor Jeame Barrueto, María Antonieta Saa D., Jaime Estévez V., presentado en 1997. Establecía un criterio de proporcionalidad en estas candidaturas, es decir, que ningún sexo superara el $60 \%$ en las listas. 2) Boletín Legislativo $\mathrm{N}^{\circ}$ 3020-06. Moción de los/as diputados/as Isabel Allende B., Aníbal Pérez L., Juan Pablo Letelier M., Carlos Jarpa W., Edgardo Riveros M., Carmen Ibáñez S., Fulvio Rossi C., Carolina Tohá M., Ximena Vidal L., de 6 de agosto de 2002. Buscó asegurar un 30\% de mujeres en las listas de candidatos/as a elecciones parlamentarias y municipales. 3) Boletín Legislativo 3206-18. Moción de los/as diputados/as Isabel Allende B., Guido Girardi L., Adriana Muñoz D., María Antonieta Saa D., Rosa González R., Carmen Ibáñez S., María Eugenia Mella G. Fulvio Rossi C., Alejandra Sepúlveda O., Carolina Tohá M., de 13 de marzo de 2003. Este proyecto corresponde a la reposición del proyecto de 1997, oportunidad en que no contó con el apoyo del Poder Ejecutivo y no se le asignó urgencia para su discusión, por lo que debió ser archivado.

8. Boletín $\mathrm{N}^{\mathrm{o}} 2608-11$.

9. Femócratas es el nombre que se ha dado a las feministas que se han incorporado a trabajar en el Estado. 
cular las complejidades de la sociedad actual. Se ha desperfilado el espacio político y hecho más difícil determinar el ámbito de la ciudadanía, puesto que la invocación de la ciudadanía contrasta con un notorio proceso de privatización (Lechner, 2002).

El liderazgo de Bachelet irrumpió, abriendo la posibilidad de continuidad de la Concertación, que parecía agotada dos o tres años atrás, superando a las figuras masculinas de los partidos, por más prometedoras que les parecían. Mezcla de cansancio con una elite que se ha repetido por 16 años los cargos en la institucionalidad pública, y necesidad de un gobierno más comprometido con la sociedad y sus percepciones subjetivas ante una modernización que la somete a crecientes tensiones, los estudios de opinión pública ya habían dado cuenta de la expectativa que había en la candidata y en sus atributos reconocidos como femeninos. No obstante, parecía una tarea imposible, considerada la larga tradición existente en el país de liderazgos y arreglos masculinos para llegar y permanecer en el poder. Los discursos electorales de las campañas de esta elección difieren considerablemente de aquellos utilizados en la elección del Presidente Lagos (Programa Mujer y Democracia en el Mercosur, 2000; Araujo, 2005). Los temas de género invadieron el debate desde una nueva perspectiva, todos los candidatos debieron incluirlos y responder a un electorado femenino exigente y empoderado.

El Programa de Gobierno de la presidenta Bachelet expresa explícitamente la voluntad de garantizar la igualdad de derechos y el ejercicio efectivo de los mismos a mujeres y hombres y considera inaceptables las desigualdades de género existentes. Entre las distintas medidas tendientes a superar dichas desigualdades, destacan aquellas que apuntan a superar la histórica marginación de las mujeres de los cargos públicos y de representación popular, medidas de acción afirmativa destinadas a restablecer los equilibrios de género como la Ley de Cuotas pensada para corregir el déficit de representación política femenina.

Al instalar de hecho la paridad en su gobierno y convocar a una Comisión asesora para el desarrollo de una propuesta de reforma del sistema electoral (Comisión Boeninger), la Presidenta ha abierto simultáneamente dos posibles debates: el de la acción afirmativa y el de la paridad, propiamente tal. Cada uno de ellos tiene fundamentos específicos, los que aún no han sido enunciados plenamente.

En efecto, la dualidad sexual del género humano, es decir, el hecho básico que todas las sociedades están conformadas por mujeres y hombres, está ausente de los debates. No se refleja en el quehacer político y social el paso desde un individuo abstracto, hacia un individuo concreto sexuado. La tensión en torno a la igualdad y la diferencia, que ha recorrido la discusión sobre la representación política de las mujeres desde fines del siglo XIX, tiene una nueva vuelta de tuerca. 
Como bien señala Bérengère Marques-Pereira, la igualdad se opone a los privilegios y a la discriminación, mientras la diferencia se opone a la semejanza, y el desarrollo democrático se ha basado en la eliminación de la lógica del privilegio en favor de la igualdad:

Mientras el privilegio recurre a la ley particular válida para algunos individuos, órdenes o sociedades, la igualdad remite a la ley general válida para todos. Mientras el privilegio legitima las diferencias en nombre de jerarquías supuestamente naturales, la igualdad ante la ley establece un espacio común que permite a los individuos relacionarse a pesar de sus particularidades. Mientras el privilegio legitima las libertades particulares ligadas al nacimiento, la igualdad de derechos remite a su universalidad, que está en el corazón de la humanidad compartida por los individuos. Mientras la lógica del privilegio descansa sobre una transformación de las diferencias en desigualdades de estatus y de condición, la igualdad ante la ley y la igualdad de trato establecen un lazo entre las particularidades constitutivas del género humano. La igualdad supone las diferencias así como el universalismo la alteridad, y así como la unicidad del género humano implica reconocer el carácter único del individuo, la mirada del otro es la mirada de un álter ego. En otras palabras, las diferencias hombres/mujeres no son vistas en el registro feudal y vertical de la inferioridad, sino en el registro democrático y de la alteridad (MarquesPereira, 2003: 125. Traducción T. Valdés).

Desde la igualdad formal frente a la ley, las políticas de los gobiernos de la Concertación han buscado la igualdad de oportunidades. Esta noción sustituye la idea de la igualdad formal y pone el énfasis en igualar los factores sociales y culturales de las situaciones de partida, bajo el supuesto de la libre concurrencia y la meritocracia en un marco de justicia distributiva. Es decir, que las desigualdades resultantes se produzcan por los distintos méritos que tengan las personas. En términos políticos, buscan garantizar la presencia pública femenina, superando la reclusión en las tareas reproductivas. Sin embargo, las políticas no han considerado la igualdad de resultados.

La noción de igualdad de resultados, es decir, de igualdad de oportunidades con aval, se funda en la idea de proporcionalidad a la que han dado consistencia las políticas de acción positiva. La igualdad de resultados no se fija ya en los casos individuales, sino que debe expresarse en resultados estadísticos y sociológicos relativos a distintos grupos sociales (Marques-Pereira, 2003). Sin embargo, también busca apoyar individualmente a las mujeres en su acceso al mundo público. Es lo que propone la CEDAW, medidas legislativas temporales a favor de las mujeres para superar las desigualdades de hecho, es decir, lograr tanto la igualdad de oportunidades como la igualdad de resultados. Sin embargo, la discriminación no desaparece en la medida en que la incorporación de las mujeres al mundo público no transforma su rol doméstico reproductivo. Ellas siguen siendo las responsables, en forma total o parcial, del ámbito familiar y doméstico, independientemente de otra actividad que 
desempeñen. La acción positiva, si bien complementa la igualdad de oportunidades, no resuelve un tema de fondo: la organización social que sustenta la discriminación de las mujeres y el rol que ocupan en la familia (Astelarra, 2004).

La tesis de la paridad, por su parte, intenta responder a una cuestión previa: ¿quién es la persona humana de la Declaración universal de 1948? ¿Cuál es el sujeto de los derechos fundamentales inalienables? El sujeto es el individuo sexuado y no el individuo abstracto. En ese sentido, la paridad es el reconocimiento de la dualidad sexual del género humano. La paridad se ubica en la perspectiva universalista, pero opone el individuo abstracto al individuo singular que es, irremediablemente, hombre o mujer. Sin embargo, reconoce al mismo tiempo una pluralidad irreductible e introduce la alteridad en la representación. En efecto, supone una refundación del pacto social que permite a las mujeres representar al universal.

\section{¿A qué apunta la categoría "género"?}

La construcción teórica de la categoría "género" es producto de distintos procesos por una parte, de las transformaciones vividas por las sociedades occidentales y su impacto en las vidas de las familias y las mujeres; en segundo lugar, de los movimientos feministas -activistas, pensadoras y académicas- y de las luchas sociales impulsadas por mujeres ${ }^{11}$; y también del desarrollo de las ideas provenientes de distintas disciplinas de las ciencias sociales y las humanidades, donde fueron tomando fuerza las ideas universales de libertad e igualdad de la Revolución Francesa.

En 1949, Simone de Beauvoir había publicado el libro "El segundo sexo", y señalado cómo el hombre había definido a la mujer como el lado oscuro de su ser, un complemento de menor valor. Acuñó entonces la famosa frase "La mujer no nace, se hace", que da cuenta de la tensión entre biología y cultura, dando un sustento filosófico y político, tanto a las luchas de las mujeres en las décadas siguientes, como al surgimiento de los "estudios de la mujer" y, posteriormente, los estudios de género.

En el ámbito académico, en los años 70 se buscó explicar la "subordinación de las mujeres", que les afecta a todas o casi todas por igual. Kate Millet (1970) recurrió entonces a la noción de "patriarcado", como cate-

10. Sigo el texto de Teresa Valdés y Heidi Fritz (2006).

11. El feminismo, como corriente filosófica y política, busca la transformación de las relaciones entre hombres y mujeres, la emancipación de las mujeres y la eliminación de su subordinación. 
goría explicativa12. Paralelamente, surgieron los "estudios de la mujer" que buscaron generar conocimientos sobre las condiciones de vida de las mujeres, sus aportes a la sociedad y la cultura, hacerlas visibles en la historia, en la creación y en la vida cotidiana (De Barbieri, 1992).

En los años 80 se comenzó a generalizar la noción de "género" como evolución posterior a la noción de "patriarcado" y el cuestionamiento del estudio aislado de las mujeres y la supuesta homogeneidad entre ellas que se apreciaba en los "estudios de la mujer". Aunque el énfasis en las mujeres se justificaba por su posición de subordinación generalizada, se planteaba la necesidad de estudiar a las mujeres en relación con los hombres y con el sistema social en que están insertas, el cual genera desigualdades y jerarquías de género. Surgieron entonces los "estudios de género".

El concepto de "género" alude a aquella construcción social y cultural (simbólica) de la diferencia biológica que hacen las sociedades. No obstante, implica un sistema de significación y acción que va mucho más allá del cuerpo. De hecho, el género constituye una de las formas de diferenciación y estratificación que las sociedades han construido. Al ser construidas socialmente, éstas se modifican, cambian y se transforman. A su vez, las construcciones culturales de género están ligadas a las construcciones que cada cultura hace de las nociones de sujeto, persona y autonomía.

En su dimensión social, la categoría "género" apunta a las relaciones sociales basadas en el género, a la división del trabajo, aquellas actividades que hacen hombres y mujeres. La dimensión cultural o simbólica remite a las representaciones de lo femenino/masculino, a las ideologías de género, los estereotipos de género, las definiciones ideales de lo que es ser hombre y ser mujer, a las valoraciones de lo femenino y lo masculino, a las identidades de género; en definitiva a las interpretaciones culturales de las prácticas, de lo que se hace.

El género se expresa a través de relaciones sociales, las cuales se constituyen de reglas, normas y prácticas, dando origen a un "sistema de sexogénero" (De Barbieri, 1992). Por medio de estas reglas se asignan recursos, tareas y responsabilidades diferenciadas, además de valoraciones según categorías de actores; al tiempo que se movilizan mecanismos y

12. Basándose en la categoría de patriarcado desarrollada por Max Weber, Kate Millet (1970) en "Política sexual", define el "patriarcado" como un sistema social basado en la dominación del sexo masculino sobre el femenino, por la fuerza o por amenaza de fuerza. Explica la subordinación femenina como producto de un orden patriarcal, en que los varones, a partir de la figura del Patriarca-Pater existente en las sociedades arcaicas bíblicas, disponían de la vida y de la muerte de hijos, esposas, esclavos y rebaños. Estima que son pocas las diferencias existentes hoy día con esas sociedades, a pesar de las apariencias. Si bien este concepto aún es utilizado en las ciencias sociales, aparece como demasiado globalizante, y por tanto, sin suficiente valor explicativo (De Barbieri, 1992). 
cuotas de poder. De modo que las desigualdades de género se reproducen a través de los distintos dominios institucionales, entre los cuales son claves la familia, el sistema educacional, las religiones, la comunidad-sociedad civil, el mercado, el orden jurídico y político y el Estado en su conjunto.

El sistema de sexo-género implica y se expresa en relaciones de poder, conceptualizado en términos procesales, relacionales y con dimensiones institucionales y estructurales, no sólo como atributo de los individuos. Se trata de un sistema jerárquico, es decir, involucra jerarquías de género, privilegios masculinos, estructuras que favorecen el dominio masculino o la valoración de lo masculino, fija límites a las mujeres y a su ubicación en determinados espacios en la sociedad. Es el caso, por ejemplo, de la existencia de reglas informales implícitas, que están favoreciendo la no toma de decisiones de las mujeres en una determinada organización, o la reclusión en el ámbito privado. En relación a la desventaja en el ejercicio del poder es que se propone la necesidad de empoderamiento como un proceso con aspectos individuales y colectivos.

La categoría "género" vincula la esfera reproductiva con la esfera productiva $^{13}$ de manera sustantiva, en la medida que la participación de hombres y mujeres en una esfera afecta su participación en la otra, así como por el hecho que las actividades desarrolladas en el ámbito reproductivo sustentan y subsidian las actividades productivas. De esta manera, los vínculos entre una esfera y otra son de carácter económico y cultural. La esfera reproductiva, al ser definida como femenina y no ser valorada ni social ni económicamente, aunque contribuya al desarrollo y la productividad de las sociedades, constituye uno de los elementos centrales en la subordinación de las mujeres.

Este orden de género articula elementos y procesos estructurales con la subjetividad de los sujetos, en tanto involucra tanto al individuo que produce e interpreta su realidad, que construye su identidad de género, como al sujeto situado en el marco de estructuras, significaciones, normas y valores de género que ordenan y modelan su comportamiento e interpretación del mundo y de los géneros.

En América Latina, los llamados "estudios de género" se desarrollaron a contar de los años 80, de la mano con la práctica feminista y la construcción de un movimiento de mujeres, como ha sido documentado por diferentes autoras (Valdés, 1993 y 1994; Hola, 1992; Kirkwood, 1986). En un

13. La esfera reproductiva identifica el trabajo que se realiza de preferencia en el hogar, que incluye las actividades relacionadas con la crianza y educación de los hijos, las labores domésticas como aseo, lavado, acarreo de agua, la preparación de alimentos, el cuidado de enfermos y/o ancianos, etc. Es un trabajo que, al ser realizado por la "madresposa" (Lagarde, 2001) en el hogar, no es remunerado (bienes y servicios con valor de uso). La esfera productiva identifica el trabajo de producción de bienes o servicios para el mercado (bienes y servicios con valor de cambio), que tienen una retribución económica. 
contexto político de dictaduras y autoritarismo, la situación de subordinación y discriminación de las mujeres se constituyó en objeto de estudio para aquellas feministas que retomaron las banderas de las luchadoras por la ciudadanía política de la primera mitad del siglo XX.

El resurgimiento de los movimientos feministas y de mujeres en la región abrió nuevas temáticas para la investigación, y los procesos de democratización inauguraron un nuevo escenario para la producción de conocimientos. Las preguntas por la ciudadanía femenina, por la ampliación de derechos en los nuevos contextos democráticos, y las posibilidades de influir en esta ampliación, se materializaron tanto en la investigación como en la acción política, nacional y regional. Esta ampliación se reflejará en la redefinición de los derechos humanos de las mujeres con la inclusión de la violencia doméstica y sexual y de los derechos sexuales y reproductivos.

\section{El aporte de la categoría género a la ciencia política}

Sin duda, la categoría género es de gran importancia para la comprensión de los procesos políticos e institucionales, el comportamiento diferencial y las prácticas de mujeres y hombres con relación a la política, la cultura política y su participación en organizaciones políticas. La construcción social de género y las relaciones de poder entre mujeres y hombres que caracterizan a cada sociedad son ineludibles para estos análisis.

En este sentido, la política está determinada por el sistema de género vigente, que la ha definido como actividad eminentemente masculina. Por otra parte, el sistema de género se mantiene y reproduce por la intervención política. Este hecho tiene dos dimensiones: si se define la política no sólo como un conjunto de actividades e instituciones, sino por sus relaciones de poder, el sistema de género también es político, porque se mantiene por el uso del poder. Pero, a su vez, el Estado interviene en la creación y mantenimiento de determinadas relaciones de género, es decir, promueve un orden de género particular. El lugar que ocupan las mujeres en la familia y la reproducción, es decir, la división sexual del trabajo, produce limitaciones para su participación en las organizaciones políticas y en las instituciones del Estado. Al dejarlas a cargo del trabajo doméstico, las deja con menos tiempo disponible a la vez que las socializa para el ámbito privado. Al mismo tiempo, la asignación de tareas por sexo dificulta que las mujeres participen en actividades consideradas masculinas. Cuando lo hacen, se les exige que extremen las características asociadas a su sexo y que sigan actuando como madres, esposas y dueñas de casa (Astelarra, 2003).

Es decir, la infrarrepresentación femenina en política -llamada también "brecha democrática" - en las democracias occidentales sólo puede ser 
plenamente explicada utilizando una "perspectiva de género"14. Este enfoque teórico permite interpretar los condicionantes y limitaciones que existen para la participación política de las mujeres, sea en las instituciones, en los partidos políticos o en las elites políticas. También la cultura política de las mujeres y las barreras que ponen los varones frente a su presencia e incorporación al mundo público en igualdad de condiciones.

La inconsistencia entre el discurso igualitario de derechos humanos con la práctica y los resultados en el ámbito político, más allá de la crítica ética, han obligado a las y los cientistas políticos/as a problematizar y explicar este fenómeno. De allí el aumento en muchos países de los estudios sobre género y política en los años 80 y 90 .

De esta forma, se han multiplicado las preguntas de investigación, abriendo un abanico temático hacia la asociatividad femenina, la acción social colectiva y la participación de las mujeres, los sistemas políticos y electorales y los mecanismos de discriminación, los partidos y las culturas políticas en su dificultad de representar los intereses de las mujeres, el comportamiento electoral femenino, la representación política de las mujeres, los intereses de las mujeres y las barreras a la participación femenina, los liderazgos políticos femeninos y masculinos, las consecuencias políticas de la baja representación femenina, la institucionalidad pública de género, las leyes de cuotas de participación por sexo y demás acciones afirmativas en el ámbito político, el impacto de la presencia de mujeres en los gobiernos y parlamentos en la agenda política, la ampliación de la ciudadanía, el/los espacio(s) público(s) y la política, las relaciones entre los ámbitos público y privado y la división sexual del trabajo, el "techo de cristal", entre otras.

Desde el punto de vista teórico, el abordaje de estas preocupaciones y temáticas ha pasado, desde la óptica conductista, donde género era sinónimo de sexo, y donde se analizaba a partir de variables dicotómicas, a un proyecto de investigación empírica donde el género es abordado como categoría y como proceso. El tránsito no ha sido fácil. Las investigadoras comprometidas con el objetivo de conocer las dinámicas a la base de las relaciones de género se han visto prontamente atrapadas por las exigencias del mainstream dominante en la disciplina. Si sus investigaciones no estaban planteadas en términos positivistas, sus posibilidades de legitimidad disciplinaria se veían seriamente comprometidas. Aun así, con esfuerzo y la complicidad que se ha ido tejiendo a través de las redes del conocimiento transnacional, hoy se visualiza el pasaje desde una preocupación original, más centrada en la pregunta ¿dónde están las mujeres?, a la interrogante acerca de ¿cuáles mujeres? Hoy, se asiste

14. "Perspectiva de género" se refiere al análisis con la categoría teórica de género, centrando la atención no sólo en las relaciones desiguales entre los géneros, sino también en las estructuras que generan y reproducen la desigualdad. Supone la inclusión de las problemáticas y especificidades de las mujeres en el reconocimiento de sus diferencias. 
paulatinamente a la instalación de una comprensión amplia del género como un fenómeno que no es simple sinónimo de sexo, sino que involucra interacciones dinámicas culturalmente específicas. En la actualidad, se entiende que las categorías de lo masculino y lo femenino no son mutuamente exclusivas, sino que más bien estarían mutuamente implicadas (Beckwith, 2005).

Desde el punto de vista metodológico, los estudios sobre género y política se encuentran en un momento de inflexión. En el contexto de un turno institucional para la Ciencia Política que se ocupa del género, se ha llegado a reconocer la necesidad de comprender la naturaleza generizada de las instituciones políticas y los procesos de cambio institucional. Una vez constatadas las limitaciones que conllevan los análisis centrados en el género a nivel individual, se reconoce que la tarea a emprender ahora es analizar las estructuras subyacentes que sostienen las ventajas y desventajas institucionalizadas. El foco se ha desplazado, hoy día, desde "mujeres en política", hacia "género y política", mediante el intento de estudiar las interacciones de hombres y mujeres como actores institucionales generizados dentro de instituciones generizadas, así como las disrupciones y contrarreacciones que se producen cuando las mujeres ingresan a espacios tradicionalmente masculinos (Mackay, 2005).

\section{Los artículos presentados en este volumen y el debate que se abre en Chile}

Los artículos que reúne este volumen dan cuenta de la riqueza de un debate conceptual y de los análisis empíricos que han surgido en la Ciencia Política occidental, al ritmo de los cambios jurídico-normativos que sitúan a las mujeres como sujetos de derechos en un plano de equidad con los varones y de los procesos socioculturales y políticos que las llevaron a romper con la histórica reclusión en el espacio privado. Se trata de una serie de trabajos de investigación sobre el espacio de las instituciones políticas, y más particularmente, sobre las prácticas políticas, procesos políticos y de institucionalización de medidas implementados para la equidad, analizados desde una perspectiva de género, casi todos escritos por cientistas políticas/os de otros países. La mayoría intenta responder a la pregunta por el déficit democrático o la subrepresentación femenina en la política y abordan experiencias y estrategias de superación de la misma. Y así como reflejan una amplitud temática, abren nuevas preguntas de investigación. De esta forma, el presente volumen de la revista Política no sólo se hace cargo de la creciente centralidad del género para la comprensión más integral de la vida política, sino que formula una invitación para que, en nuestro contexto, no se reproduzcan las condiciones de marginalidad, cuando no de aislamiento, que estas preocupaciones y sus cultoras han experimentado en otras comunidades disciplinarias en el mundo. 
Los artículos del presente número abordan seis temáticas: a) los marcos de análisis y el estado del arte sobre la posición de las mujeres en el proceso de toma de decisiones políticas; b) las leyes de cuotas y de paridad con los argumentos de los partidos al respecto, las experiencias por países, las condicionantes para su eficacia así como sus consecuencias en las prácticas políticas; c) las estrategias de los partidos políticos para promover la participación femenina y sus prácticas para eludir las obligaciones partidarias; d) las estrategias políticas feministas y la agenda pública en Chile; e) el impacto de las mujeres en los órganos legislativos y en los productos de la política, y f) las relaciones sociales de sexo en el desarrollo de movimientos sociales de cesantes (formación, irrupción, divisiones, extinción).

\section{a) Los marcos de análisis:}

Marta Lois e Isabel Diz, en el artículo titulado “¿Qué sabemos de la presencia política de las mujeres y la toma de decisiones? Claves para un marco de análisis", hacen una completa revisión, desde una doble perspectiva, normativa y politológica, de los principales argumentos y orientaciones acerca de la presencia de las mujeres en el ámbito de la política, con la idea de establecer las claves para un posible marco de análisis. Este artículo permite situar al lector en el panorama más amplio de la posición de las mujeres en los lugares donde se toman las decisiones vinculantes para la sociedad, con particular énfasis en el Poder Legislativo. Un ámbito interesante abordado es el de los análisis de la administración pública y la noción de "burocracia representativa", según la cual las administraciones deben ser representativas de los ciudadanos a los que sirven. En sus conclusiones, resaltan los desafíos que se presentan en los ámbitos donde se instalan los nudos gordianos a desatar para superar la mera retórica: la doble carga laboral y familiar para las mujeres, el modelo masculino de vida política y los roles sociales predeterminados asignados a las mujeres.

b) Las medidas para el fomento de las mujeres en la vida política: leyes de cuota y paridad (debates, experiencias, eficacia y barreras).

Jutta Marx, Jutta Borner y Mariana Caminotti analizan en "Cuotas de género y acceso femenino al Parlamento: los casos de Argentina y Brasil en perspectiva comparada" dos países que cuentan con una ley de cuotas de género. Señalan las características del sistema electoral y de la ley en cada país y los resultados obtenidos en cada caso, junto con los factores que condicionan la eficacia de las cuotas en dichos contextos. Examinan en qué medida el éxito de las cuotas de género depende de las características del sistema electoral, de la precisión de las normas que sustentan este tipo de medidas y de la participación de los actores nacio- 
nales en su instrumentación y vigilancia por su correcta aplicación. Analizan el impacto que tiene el hecho que la ley cuente o no con sanciones expresas que penalicen el incumplimiento de los requisitos previstos en la legislación por parte de los partidos, así como la importancia que tiene el control de las organizaciones de mujeres y las aspirantes a candidaturas para que dicha legislación se cumpla. Sus hallazgos no son menores por cuanto identifican los elementos que permiten que una ley de cupos femeninos sea realmente efectiva.

Por su parte, Delphine Dulong y Frédérique Matonti analizan, en el artículo titulado "Reproducción dentro de la revolución: género y mujeres frente a la ley francesa de paridad en política", lo que ha sucedido en Francia después de que el gobierno de Lionel Jospin sometió a votación, el 6 de junio de 2000, una ley para favorecer el acceso igualitario de mujeres y hombres a los mandatos electorales y a las funciones electivas. Más ambicioso que las leyes de cuotas, organiza la paridad de representación para todos los escrutinios de listas. Los partidos están obligados a poner tantas mujeres como hombres en los primeros seis lugares de las listas electorales. Se prevén sanciones financieras para los partidos que no colocan tantas mujeres como hombres.

Las autoras consideran que, de acuerdo a los resultados electorales, la revolución esperada por las feministas no se produjo, que donde la paridad es obligatoria por ley, hay una feminización de los cargos elegidos, pero no en los otros casos. Para ellas, la ley paritaria no resiste las reglas tácitas del campo político francés que es muy cerrado frente a las lógicas del orden social y, particularmente, a los movimientos sociales. Ello, porque si se admite que la actividad política tiene por objeto central la conquista y conservación del poder, no hay que extrañarse de que los profesionales de la política hayan intentado limitar las consecuencias de la ley sobre su propia carrera. La regla paritaria pone en cuestión la economía de las retribuciones de la militancia, al tiempo que hace más complejos los equilibrios intrapartidarios y territoriales en la confección de las listas, reduce los puestos a ofrecer a los militantes de sexo masculino, que son más numerosos que las mujeres. A ello se suma el lugar poco reconocido que tiene el feminismo en la política, donde incluso resulta ser estigmatizante. Las mujeres políticas tienden a renunciar al interés de género por el interés de clase.

c) Los partidos politicos: sus estrategias para promover la participación femenina y sus prácticas para eludir las obligaciones partidarias

El trabajo de Tània Verge titulado "De la cuota a la democracia paritaria: estrategias partidistas y representación política de las mujeres en España" se concentra en los discursos y prácticas de los diferentes partidos en torno a la paridad. La autora analiza los factores que han 
contribuido a que la democracia española supere a democracias más antiguas y consolidadas en cuanto a la representación política de las mujeres. Para ello, se examinan las estrategias desarrolladas por los tres partidos de ámbito nacional en los tres niveles planteados por Lovenduski y Norris (1993) según los cuales los partidos pueden adoptar estrategias retóricas, de acción o de discriminación positiva para alentar una mayor participación femenina.

Desde otra perspectiva, David Garibay analiza la problemática de género y su posible uso instrumental a partir del estudio de un partido político de izquierda, con origen revolucionario. En el caso del FMLN, de El Salvador, la adopción de una perspectiva de género dentro de sus estatutos y su programa permite expresar la radicalidad del partido. Sin embargo, este compromiso se estanca por cuanto las mujeres no han logrado avanzar significativamente en las estructuras de dirección y de representación del partido.

Ambos estudios son altamente pertinentes por cuanto toda la literatura insiste en señalar el rol protagónico de los partidos políticos a la hora de estructurar las oportunidades políticas de las mujeres.

\section{d) Las estrategias politicas feministas y la agenda pública en Chile}

Liesl Haas expone las reglas del juego que enfrenta lo que ella denomina "la política feminista" en Chile, en el artículo titulado "The Rules of the Game: Feminist Policymaking in Chile". Analiza los avances alcanzados por las mujeres desde la vuelta a la democracia con aquellos cambios legislativos que apuntan a los objetivos del movimiento feminista. Ello, en un contexto de sensibilidad de los gobiernos chilenos a las demandas ciudadanas por igual trato, como tarea central de la democratización.

El balance legislativo de la agenda política feminista revela que, en 16 años, se han presentado al Parlamento 42 propuestas legislativas en un amplio abanico de temas, la mayoría, por iniciativa parlamentaria. La mayor eficacia ha estado en los proyectos presentados por el Sernam (8 de los 9), mientras sólo 8 de las 38 iniciativas de origen parlamentario fueron aprobadas entre 1990 y 2001. Sin embargo, todas estas propuestas han sufrido cambios importantes durante el debate, perdiendo a veces su carácter feminista. En ello han influido tanto la rigidez y naturaleza de la política chilena por la sobre-representación de los sectores conservadores, como el aprendizaje de las feministas en cuanto a moverse en las arenas institucionales y culturales. Según la autora, las parlamentarias han debido balancear su lealtad hacia sus partidos políticos con el deseo de avanzar en la agenda feminista, cuando ésta genera controversia.

El sistema político condiciona en efecto la acción de las parlamentarias, que deben buscar bases amplias de apoyo, moderar el lenguaje, el carácter 
de las propuestas, obtener el respaldo de todos los sectores de la coalición, consensuar y aceptar progresos graduales antes que cambios radicales.

La autora analiza tres casos: la ley que penaliza la violencia doméstica, la ley de matrimonio civil y las propuestas de despenalización del aborto, considerando la significación de los proyectos presentados, el marco estratégico y de negociación establecido en cada caso, la existencia o no de cooperación institucional y las lecciones aprendidas.

e) El impacto de las mujeres en los órganos legislativos y en los productos de la política

Niki Johnson, a partir del caso de la XLV legislatura uruguaya, examina si se pueden identificar diferencias de género en la actividad parlamentaria de legisladores/as y hasta qué punto las parlamentarias juegan un rol específico en la promoción de iniciativas de género. Su artículo se titula "Actividad parlamentaria y diferencias de género en Uruguay". El tema que aborda es de gran interés en la literatura por cuanto existe una permanente preocupación por saber si las mujeres, cuando ingresan a la arena política, hacen la diferencia. La comparación de actividades parlamentarias muestra que las legisladoras eran más prolíficas que sus pares varones. Aunque en ambos casos se producía un patrón tradicional de la división sexual del trabajo en el enfoque temático de su quehacer parlamentario, en el caso de las mujeres este sesgo generalmente era menos marcado y, en algunos casos, hasta se invertía. Asimismo, analiza los factores que permiten que la pequeña minoría de legisladoras logre transformarse en una masa crítica que promueva una agenda de género, a través de la bancada femenina intrapartidaria.

\section{f) Las relaciones sociales de sexo en movimientos sociales de cesantes}

Desde la tradición sociológica francesa, Xavier Dunezat examina, a partir de un estudio de caso, las "relaciones sociales de sexo" en dos movimientos sociales de cesantes en su artículo "Luchas dentro de la lucha. Acción colectiva y relaciones sociales de sexo". Más allá de los avances de los estudios de los movimientos sociales, que consideran la formación de la acción colectiva, el paso de la movilización individual a la colectiva, las relaciones entre la acción colectiva y su entorno (los medios, las estructuras de oportunidades políticas, el campo poliorganizacional, los movimientos contrarios) y las dimensiones simbólicas de la movilización (enfoque, alineación de marcos interpretativos de la realidad, producción de identificaciones), se interesa en el paso desde la movilización colectiva a la salida individual, en la variabilidad de las acciones colectivas dentro de un mismo movimiento, en lo vivido por el grupo de movilizados, tanto en términos de representaciones como de prácticas. 
Cruzando la sociología de los movimientos sociales con la sociología de las relaciones de sexo, examina la forma en que las relaciones sociales de sexo impregnan un movimiento social en los diferentes momentos de su historia. Al considerar el movimiento social como sexuado, hace visible la existencia de grupos de sexo construidos socialmente -hombres y mujeres- que están atravesados por relaciones de poder. A partir de la observación participante, analiza lo que hacen los movilizados en el movimiento -qué se hace y quién lo hace- y da cuenta de una diversidad de formas de participación y de compromisos organizacionales (participación continua, alternada, reservada).

Este trabajo levanta, para Chile, numerosas interrogantes a la reciente movilización de estudiantes secundarios y su liderazgo rigurosamente paritario.

En la sección "Debates y Reseñas" se incluye el artículo de Ma. de los Ángeles Fernández quien, en el marco de la autorreflexión de la Ciencia Política, sitúa la producción académica sobre género y política de los / as cientistas políticos chilenos, la describe e intenta explicar las razones de su pobreza y presencia minoritaria. Presenta un interesante panorama de la actividad académica en este campo temático, en la docencia y en las publicaciones, e interpela a la Ciencia Política local acerca de la necesidad de aprovechar la actual oportunidad política y disciplinaria para incorporar ampliamente los estudios sobre género y política, en sus más complejas dimensiones.

En general, todos los artículos incluidos en el presente dossier No 46 de la Revista Política son iluminadores para el debate que se ha abierto en Chile. Ello, porque si bien la elección de Michelle Bachelet como Presidenta de la República y el nombramiento de un gabinete ministerial y de gobiernos regionales paritarios sitúa a Chile a nivel de los países con mayores niveles de equidad de género en el ámbito gubernamental, la paridad recién se instala en el horizonte de análisis de muchos investigadores.

No obstante, es necesario admitir que la situación que hoy se observa en el ámbito del Poder Ejecutivo, por la voluntad política de la Presidenta, está lejos de la que se aprecia en los demás poderes del Estado, muy particularmente, del Poder Legislativo. En efecto, nuestro país muestra cifras pobres en cuanto al número de diputadas y senadoras, cargos representativos de elección popular, en cuyo acceso los partidos políticos juegan un rol trascendental al proponer a la ciudadanía candidatos o candidatas (ver Anexo 2). En este marco se ha reabierto la discusión sobre la ley de cuotas que se había dado anteriormente sin mayores frutos, en la medida en que se consideraba inviable con el sistema electoral binominal vigente (Núñez, 2001; Stoffel, 2005; Veloso, 2005).

En ese sentido, los debates de otros países que incluyen los artículos de esta revista no difieren de los que se vuelven a escuchar hoy en Chile, y 
los argumentos esgrimidos en esos lugares por actores de todo el espectro político bien podrían ser de los actores nacionales, especialmente aquellos de los sectores conservadores. Sin embargo, abren una panorámica mayor por cuanto involucran una evaluación de la aplicación de leyes de cuota y de paridad con una perspectiva de tiempo mayor: España, Argentina, Brasil, Francia constituyen ejemplos que anticipan el tipo de desarrollo que pueden tener en Chile el debate discursivo y la práctica política en un escenario que recién se insinúa.

Son relevantes también en cuanto presentan actores sociales y políticos, algunos de los cuales siguen libretos tradicionales en términos del orden de género, mientras otros desarrollan estrategias de cambio. No obstante, se trata de trabajos todavía iniciales, que revelan un campo de análisis en construcción, que deben dialogar con mayor profundidad con otras disciplinas de las ciencias sociales y, de manera especial, con la teoría política por ser éste el ámbito que se ha abierto, con mayor generosidad, al diálogo con las preocupaciones provenientes del feminismo.

\section{Bibliografía}

Araujo, Kathia. 2005. "Significations et représentations imaginaires relatives aux femmes dans l'espace politique chilien (1999-2003)". En Représentation politique des femmes au Chili. Cahiers du GELA-IS No 5. Belgique: GELA-IS, Université libre d Bruxelles - L'Harmattan, 159-186.

Astelarra, Judith. 2003. ¿Libres e iguales? Sociedad y politica desde el feminismo. Santiago: CEM Ediciones.

2004. Políticas de género en la Unión Europea y algunos apuntes sobre América Latina. Serie Mujer y Desarrollo No 57. Santiago: CEPAL.

Bareiro, Line, et al., 2004. Sistemas electorales y representación femenina en América Latina. Serie Mujer y Desarrollo $N^{\circ} 54$. Santiago: CEPAL.

Beckwith, Karen. 2005. "A common language of gender?". Politics \& Gender 1 (1): 128-137.

\section{CEPAL. 2005. Panorama Social de América Latina. Santiago: CEPAL.}

CEPAL. 2005. Balance preliminar de las economías de América Latina y el Caribe. Santiago: CEPAL.

Claro, Magdalena. 2005. Acción afirmativa. Hacia democracias inclusivas. Santiago: Fundación Equitas.

De Barbieri, Teresita. 1992. "Sobre la categoría de Género. Una introducción teórico metodológica". En ISIS Fin de Siglo, Género y Cambio civilizatorio. Ediciones de las mujeres $\mathrm{N}^{\circ}$ 17. Santiago de Chile, 111-128.

Eisler, Riane. 1990. El Cáliz y la Espada. Santiago: Cuatro Vientos.

FLACSO - Grupo Iniciativa Mujeres. 2006. Índice de Compromiso Cumplido para la Equidad de Género - ICC. Chile 1995-2003. Santiago: FLACSO. 
Frohmann, Alicia; Valdés, Teresa. 1995. "Democracy in the Country and in the Home: The Women's Movement in Chile". En Amrita Basu (ed.), The Challenge of Local Feminisms. Women's Movements in Global Perspective. USA: Westview Press, 276-301.

Gimbutas, Marija. 1995. The Language of the Goddess San Francisco: Harper.

Hola, Eugenia 1991. "El estado de la investigación feminista en Chile", Documento de Trabajo, Santiago: CEM.

Jelin, Elizabeth. 1997. "Igualdad y diferencia: dilema de la ciudadanía de las mujeres en América Latina". Cuadernos de Estudios Políticos 7: 189-213.

. 1996. “Las construcción de la ciudadanía: entre la solidaridad y la responsabilidad". En Jelin, Elizabeth y Eric Herschberg (coords.), Construir la democracia: derechos humanos, ciudadanía y sociedad en América Latina. Venezuela: Editorial Nueva Sociedad, 113-131.

Kirkwood, Julieta. 1986. Ser política en Chile: Las feministas y los partidos. Santiago: FLACSO.

Lamas, Marta. 2000a. "La antropología feminista y la categoría 'género'". En Lamas, M. (comp.), El género: construcción cultural de la diferencia sexual. México D.F.: PUEG, UNAM, 97-125.

. 2000b. "Usos, dificultades y posibilidades de la categoría 'género'". En Lamas, M. (comp.) El género: construcción cultural de la diferencia sexual. México D.F.: PUEG, UNAM, 327-366.

Lagarde, Marcela. 2001. Los cautiverios de las mujeres: madresposas, monjas, putas, presas y locas. México: UNAM, PUEG.

Lechner, Norbert. 2002. Las sombras del mañana. La dimensión subjetiva de la política. Santiago: LOM.

Lerner, Gerda. 1986. The Creation of Patriarchy. New York: Oxford University Press.

León, Magdalena (ed.). 2005. Nadando contra la corriente. Mujeres y cuotas políticas en los paises andinos. Bogotá: UNIFEM, UNFPA, Universidad Nacional de Colombia, IEP, CIDEM, FLACSO.

Lycklama à Nijeholt, Geertjé, et al. (comps.). 1996. Triángulo de poder. Bogotá: TM Editores.

Mackay, Fiona. 2005. "Gender and political representation in the UK: the state of the discipline". BJPIR (6): 99-120.

Marques-Pereira, Bérengère. 2003. La citoyenneté politique des femmes. Paris: Armand Colin.

MIDEPLAN. 2003. Encuesta CASEN.

Miles, Rosalind. 1988. Who Cooked the Last Supper: The Women's History of the World. New York: Three Rivers Press.

Millet, Kate. 1995. Política Sexual. Madrid: Cátedra.

Montecino, Sonia, et al., (eds). 1990. Tramas para un nuevo destino. Propuestas de la Concertación de Mujeres por la Democracia. Santiago: s/e. 
Mouffe, Chantal. 1997. Liberalismo, pluralismo y ciudadanía democrática. México: IFE. $h t t p: / / w w w . b i b l i o j u r i d i c a . o r g / l i b r o s / l i b r o . h t m ? l=488$

Norris, Pippa. 1997. "Las mujeres políticas: ¿un nuevo estilo de liderazgo?". En Mujeres en política (Uriarte, Edurne y Arantxa Elizondo, eds.). Barcelona: Editorial Ariel, 53-75.

Núñez, Sonia. 2001. "La représentation politique des femmes au Chili”. En Marques-Pereira, Bérengère y P. Nolasco (coords), La représentation politique des femmes en Amérique latine. Bélgica: GELA-IS, 111-138.

Organización de las Naciones Unidas. 2000. Declaración del Milenio. Resolución aprobada por la Asamblea General (A/55/L.2)] 55/2. http://www.un.org/spanish/ millenniumgoals/ares552.html

ONU. 1979. Convención sobre la Eliminación de todas las formas de discriminación contra la mujer. $h t t p: / / u n d p . o r g / r b l a c / g e n d e r / c a m p a i g n-s p a n i s h / c e d a w . h t m$

Palestro, Sandra. 1991. "Mujeres en movimiento. 1973-1989". Serie Estudios Sociales $\mathrm{N}^{\circ}$ 14. Santiago: FLACSO.

Programa Mujer y Democracia en el MERCOSUR. 2000. Silencios y susurros. Ciudadanía y género en las campañas presidenciales de Argentina, Chile y Uruguay. Santiago: Programa Mujer y Democracia en el MERCOSUR.

Rubin, Gayle. 2000. “El tráfico de mujeres. Notas sobre una Economía Política del sexo". En Lamas, Marta (comp.), El género: la construcción cultural de la diferencia sexual. México: PUEG, 35-96.

Scott, Joan. 2000. "El género: una categoría útil para el análisis histórico". En Lamas, M. (comp.), El género: construcción cultural de la diferencia sexual. México: PUEG, UNAM, 265-302.

Stoffel, Sophie. 2005. "Le débat chilien sur la représentation politique des femmes el sur le projet d'une 'lois des quotas'". En Cahiers du GELA-IS N ${ }^{\circ} 5$. Belgique: GELA-IS, Université libre d Bruxelles - L'Harmattan, 21-49.

Valdés, Teresa; Muñoz, Ana María; Donoso, Alina. 2005. 1995-2003: ¿Han avanzado las mujeres? Índice de Compromiso Cumplido Latinoamericano. Santiago: FLACSO-UNIFEM.

Valdés, Teresa (coord.); Caro, Pamela; Faúndez, Alejandra; Guerrero, Elizabeth; Muñoz, Ana María; Palacios, Indira; Valdés, Ximena. 2001. El Índice de Compromiso Cumplido - ICC. Una estrategia para el control ciudadano de la equidad de género. Santiago: FLACSO.

Valdés, Teresa. 2000. De lo social a lo político. La acción de las mujeres latinoamericanas. Santiago: FLACSO, LOM.

1993. "El movimiento social de mujeres y la producción de conocimientos sobre la condición de la mujer", Documento de Trabajo FLACSO, Serie Estudios Sociales $N^{\circ} 43$. Santiago de Chile: FLACSO.

1994. "Movimiento de mujeres y producción de conocimientos de género: Chile 1978-1989". En León, Magdalena (comp.), Mujeres y participación política: avances y desafíos en América Latina. Bogotá: T.M. Editores, 291-318.

Valdés, Teresa; Gomáriz, Enrique (coords). 1995. Mujeres Latinoamericanas en Cifras. Tomo comparativo. Santiago: FLACSO, Instituto de la Mujer (España). 
1992. “Mujeres Latinoamericanas en Cifras. Avances de Investigación. Chile. VIII. Organismos y acción de promoción de la mujer", Documento de Trabajo FLACSO, Serie Estudios Sociales No 37, Santiago de Chile: FLACSO.

1991. “Mujeres Latinoamericanas en Cifras. Avances de Investigación. Chile. VII. Participación socio-política", Documento de Trabajo FLACSO, Serie Estudios Sociales No 19. Santiago de Chile: FLACSO.

Veloso, Paulina. 2005. “Étude sur la faisabilité d'une loi de quotas dans l'ordre juridique chilien". Cahiers du GELA-IS N ${ }^{\circ}$ 5. En Représentation politique des femmes au Chili. Belgique: GELA-IS, Université libre de Bruxelles L'Harmattan, 75-104.

Zavala, Ximena. 2005. "Participation et représentation politique des femmes: un défi au quotidien". Cahiers du GELA-IS N ${ }^{\circ} 5$. En Representation politique des femmes au Chili. Belgique: GELA-IS, Université libre de Bruxelles - L'Harmattan, 51-73. 


\section{ANEXO 1}

Leyes aprobadas entre 1991-2005

- Ley $\mathrm{N}^{\circ}$ 20.086, modifica la Ley $\mathrm{N}^{\circ} 19.968$ de Tribunales de Familia (2005)

- Ley $\mathrm{N}^{\mathrm{o}} 20.066$ de Violencia Intrafamiliar, que modifica la Ley $\mathrm{N}^{\circ}$ 19.325 aprobada en 1994 (2005)

- Ley No 20.047, establece el permiso paternal en el Código del Trabajo (2005)

- Ley N 20.030 de Filiación, modifica la Ley No 19.585 de 1999 (2005)

- Ley No 20.005, tipifica y sanciona el Acoso Sexual (2005)

- Ley No 19.968, crea los Tribunales de Familia (2005)

- Ley N 19.947 de Matrimonio Civil, que sustituye la Ley vigente desde el año 1884 (2004)

- Ley $\mathrm{N}^{0} 19.852$ sobre Subrogación para las mujeres alcaldes en el período pre y postnatal (2003)

- Ley $\mathrm{N}^{\circ}$ 19.824, modifica el artículo 203 del Código del Trabajo, disponiendo la obligatoriedad de instalar salas cunas en establecimientos industriales y de servicios que indica (2002)

- Ley $\mathrm{N}^{\circ}$ 19.749, facilita la creación de microempresas familiares (2002)

- Ley $\mathrm{N}^{\circ} 19.741$ sobre Abandono de Familia y Pago de Pensiones Alimenticias (2001)

- Ley $\mathrm{N}^{0}$ 19.711, regula el derecho a visita a los hijos sometidos a la tuición de uno de los padres (2001)

- Ley $\mathrm{N}^{\circ} 19.688$, modifica la Ley $\mathrm{N}^{\mathrm{0}} 18.962$, orgánica constitucional de enseñanza, en lo relativo al derecho de las estudiantes que se encuentren embarazadas o que sean madres lactantes de acceder a los establecimientos educacionales (2000)

- Ley $\mathrm{N}^{\circ} 19.670$, extiende el beneficio del fuero a mujeres y hombres que adoptan un hijo en conformidad a la ley de adopción (2000)

- Ley $\mathrm{N}^{0}$ 19.617, modifica la tipificación de ciertos delitos sexuales incluyendo violación, violación conyugal, estupro, incesto, abuso sexual de menores y pornografía con menores (1999)

- Ley $\mathrm{N}^{\circ}$ 19.611, reforma art. 1 y 19 Nº 2 de la Constitución Política del Estado señalando de "las personas" nacen libres e iguales en dignidad y derechos. Establece también que "hombres y mujeres son iguales ante la ley" (1999) 
- Convenio de la OIT $\mathrm{N}^{\mathrm{o}} 103$ sobre protección a la maternidad ratificado y $\mathrm{N}^{\circ} 156$ sobre igualdad de oportunidades y de trato a trabajadoras y trabajadores con responsabilidades familiares, ratificados mediante Decreto 1.907 (1999)

- El Estado chileno ratifica la Convención Interamericana para Prevenir, Sancionar y Erradicar la Violencia contra la Mujer (Convención de Belém do Pará, aprobada en 1994 por la Asamblea General de la OEA) (1998)

- Ley $\mathrm{N}^{0}$ 19.591, prohíbe el test de embarazo como exigencia para ser contratada, promovida o mantenida en un empleo y concede fuero maternal a la trabajadora de casa particular (1998)

- Ley $\mathrm{N}^{0}$ 19.585, modifica el Código Civil en materia de Filiación, reconoce la igualdad jurídica de los hijos nacidos dentro y fuera del matrimonio, posibilita la investigación de la paternidad o maternidad, amplía la patria potestad de la madre y mejora la situación hereditaria del cónyuge superviviente (1998)

- Ley $\mathrm{N}^{\circ}$ 19.532, establece la jornada escolar completa (1997)

- Ley $\mathrm{N}^{\circ} 19.505$, otorga permiso especial a trabajadores en caso de enfermedad grave de hijo mayor de un año y menor de 18, aplicable a la madre o el padre (1997)

- Dictamen 225-85 de la Dirección del Trabajo, otorga el Derecho a Sala Cuna para las trabajadoras de Centros Comerciales con una misma personalidad jurídica (1996)

- Ley $\mathrm{N}^{\mathrm{0}}$ 19.482, modifica artículo 38 del Código del Trabajo en materia de descanso dominical (1996)

- Ley No 19.409, crea el delito de Tráfico de Personas (1995)

- Ley N 19.335 de Régimen de Participación en los Gananciales, que modifica diversos textos legales discriminatorios hacia las mujeres en los Códigos Civil y Penal, como el adulterio (1994)

- Ley $N^{0} 19.325$ de Violencia Intrafamiliar (1994)

- Ley $\mathrm{N}^{0} 19.299$ que modifica la base de cálculo del subsidio maternal (1994)

- Ley $\mathrm{N}^{\mathrm{o}}$ 19.250, modifica el Contrato Individual de Trabajo y la protección a trabajadoras y trabajadores (1993)

- Ley No 19.023, crea el Servicio Nacional de la Mujer (SERNAM) (1991) 
ANEXO 2

Indicadores sociales y de participación política de las mujeres en Chile

a) Indicadores sociales

\begin{tabular}{|c|c|c|c|}
\hline & 1995 & 2000 & 2003 \\
\hline Analfabetismo $(\%)$ & 5,3 & 4,4 & 3,6 \\
\hline $\begin{array}{l}\text { Años de estudio de la población económica activa femenina } \\
\text { de } 15 \text { años y más urbana (número de años) }\end{array}$ & 10,9 & 11,4 & 11,6 \\
\hline Tasa de fecundidad (número de hijos por mujer) & 2,5 & 2,4 & 2,2 \\
\hline Participación económica de las mujeres urbanas (\%) & 39,0 & 42,0 & 45,0 \\
\hline Mujeres en empleo doméstico (\%) & 16,5 & 16,0 & 16,3 \\
\hline Desempleo abierto femenino urbano $(\%)$ & 8,4 & 11,2 & 12,4 \\
\hline Desempleo abierto de jóvenes urbanas de 15-24 años (\%) & 19,3 & 23,7 & 26,3 \\
\hline $\begin{array}{l}\text { Mujeres asalariadas con contrato de trabajo respecto del total } \\
\text { de mujeres asalariadas }(\%)\end{array}$ & 76,1 & 72,3 & 73,7 \\
\hline $\begin{array}{l}\text { Relación entre salarios medios de mujeres y hombres con } \\
13 \text { años y más de instrucción, en zonas urbanas }(\%)\end{array}$ & 58,0 & 60,0 & 65,0 \\
\hline $\begin{array}{l}\text { Ingreso medio por trabajo de las mujeres en comparación } \\
\text { con los hombres }(\%)\end{array}$ & 67,0 & 65,0 & 64,0 \\
\hline Hogares con jefa mujer & 22,4 & 24,3 & 27,1 \\
\hline
\end{tabular}

Fuentes: MIDEPLAN (CASEN), CEPAL 2005, SERNAM, FLACSO 2006 (ICC).

b) Indicadores de participación política (en porcentajes)

\begin{tabular}{lrrrr}
\hline & $\mathbf{1 9 9 5}$ & $\mathbf{2 0 0 0}$ & $\mathbf{2 0 0 3}$ & $\mathbf{2 0 0 6}$ \\
\hline Mujeres en cargos de Ministras & 15,8 & 31,25 & 16,67 & 50,0 \\
Mujeres en cargos de Subsecretarias & 3,6 & 25,9 & 27,6 & 48,4 \\
Mujeres en cargos de SEREMIS & 7,6 & 22,9 & 15,3 & 38,4 \\
Mujeres en cargos de Intendentas & 7,7 & 23,1 & 15,4 & 50,0 \\
Mujeres en cargos de gobernadoras & 10,0 & 30,0 & 24,0 & 33,3 \\
Mujeres en cargos de la Cortes de Apelaciones & 27,5 & 34,0 & 35,0 & $\mathrm{n} / \mathrm{d}$ \\
Mujeres en cargos de Diputadas & 7,5 & 11,7 & 12,5 & 15,8 \\
Mujeres en cargos de Senadoras* & 2,6 & 5,2 & 5,2 & 5,2 \\
Mujeres en cargos de Alcaldesas & 7,2 & 7,9 & 12,6 & 12,2 \\
Mujeres en cargos de Concejalas & 12,5 & 17,0 & 17,0 & 21,0 \\
Mujeres en cargos nacionales de Partidos Políticos & 9,0 & 17,2 & 18,5 & $\mathrm{n} / \mathrm{d}$ \\
\hline
\end{tabular}

* Se consideran sólo los cargos de senadores elegidos democráticamente.

$\mathrm{n} / \mathrm{d}$ : No hay datos disponibles.

Fuentes: FLACSO (ICC), SERNAM, Servicio Electoral, Guía Silber, Corporación Humanas. 
$\perp^{\prime}$

$1 L$

$\overline{7}$

$\mid \overline{\bar{F}}$ 